\title{
Genotype and Phenotype Correlation of Breast Cancer in BRCA Mutation Carriers and Non-Carriers
}

Salha Mohammed bujassoum AL Bader, Hekmet Abubaker Bugrein* and Reem Jawad Al-Sulaiman

Medical Oncology, Hereditary Cancer National Center for Cancer Care and Research, NCCCR, ALDoha, Qatar

\begin{abstract}
Introduction: Breast cancer is both genetically and histopathologically heterogeneous disease. The biological basis for this heterogeneity is unknown, although there are some distinct phenotype-genotype correlations. Approximately $5 \%$ to $10 \%$ of breast cancer is hereditary and BRCA1 and BRCA2 genes are responsible for the majority of the hereditary cases of breast cancer. According to the existing literature BRCA1 and BRCA2 associated breast cancer, it has been shown that BRCA associated breast cancers have different clinical, histological and immune-phenotypic features. To validate the effect of BRCA 1 or BRCA 2 germ line mutations on breast cancer aggressiveness and its impact on breast cancer clinical and histological features, we compared the histological, molecular status and clinical variables of 32 breast cancer patients with $B R C A$ gene pathogenic mutations carriers and to the histopathological and molecular characteristics of 50 patients affected with breast cancer in the same age group but with no pathogenic mutations or variants of unknown significant (VUS) in either BRCA1 or BRCA2 genes.
\end{abstract}

Methods: A retrospective study was conducted to study breast cancer cases that were evaluated at the hereditary breast and ovarian cancer clinic at the national center of cancer care and research (NCCCR) in the State of Qatar from 2013 to 2015 . Review of medical records was conducted to determine the clinical characteristics, the molecular results of BRCA testing and the tumor characteristics from the histopathology reports in addition to a new review of the tumor blocks to update the molecular data of those patients.

Results: (82) patients with breast cancer were diagnosed at a young age (50 years of age and younger). (50) patients were BRCA negative and (32) patients were BRCA positive. (22) Patients were found to have BRCA1 pathogenic mutations, (9) patients had BRCA2 pathogenic mutations and (1) patient carried pathogenic mutations in both BRCA1 and BRCA2 genes. Most patients had mutations in the BRCA1 with most mutations being small frame shift deletion or insertion in one or more exons that caused protein truncation. BRCA mutations was detected among women of younger ages. In terms of histopathology, the majority of BRCA associated breast cancers are invasive ductal carcinoma (IDC) detected in $94 \%$ with (74\%) high nuclear grade $3 / 3$, have a higher number of mitosis and show a high frequency of necrotic areas, and a higher proliferative rate and lymphocytic infiltration. All of these histopathological features point toward a more aggressive tumor type. In terms of immunohistochemical (IHC) tumor markers, triple negative detected in $56.3 \%$ accounted for most of the BRCA associated breast cancers. Triple positive accounted for $9.4 \%$, followed by ER PR positive Her2 negative (34.4\%) and ER PR negative Her2 positive $(0 \%)$. Disease staging of all patients with breast cancer is done according to AJCC staging manual. Patients with BRCA pathogenic mutations diagnosed with stage II $60 \%$ followed by stage III $26.6 \%$ and stage IV $14 \%$. Patients with no pathogenic mutations in the BRCA genes also had IDC as being the most common histopathologcal type IDC $58 \%$ but also exhibited other type of histopathology such as ductal carcinoma in situ (DCIS) (10\%), invasive lobular carcinoma (ILC) (6\%), lobular carcinoma in situ (LCI). BRCA negative breast cancers exhibited $2 \%$ nuclear grade $3 / 3$ in $36 \%$ of cases and high proliferative rate in $31 \%$. Triple negative breast cancers accounted for $17 \%$, triple positive 13\%, ER PR positive Her2 negative $44 \%$ and ER PR negative Her 2 positive $13 \%$. In terms of disease stage, those with no pathogenic mutations in the BRCA genes mostly had stage I $39 \%$ followed by stage II $61 \%$, stag III $29 \%$, and no cases were diagnosed with stage IV.

Conclusion: These results suggest that breast cancers associated with BRCA mutations are more likely to be basal sub-type and exhibits more aggressive behavior, particularly in younger age groups and those patients present with a more advanced stage of disease than those with no pathogenic mutations in the BRCA genes who exhibit a less aggressive disease. We will continue to build our database for better characterization of our hereditary breast cancer cases at a clinical and molecular levels and use this information for future development of targeted anti-cancer agents use.

Keywords: Breast cancer; Hereditary breast cancer; BRCA genes; Triple negative; Hormonal receptors; Her2neu stage

\section{Introduction}

Breast cancer is the most common malignant disease and the second leading cause of cancer death among women [1,2]. Most women with breast cancer have a sporadic rather than an inherited breast cancer [2]. Early onset at diagnosis and family history of breast cancer are generally considered indicators of genetic susceptibility to breast cancer. Several studies have shown that early onset breast cancer displays histological features of aggressive cancer phenotype that have
*Corresponding author: Hekmet Abubaker Bugrein, Medical Oncology, Hereditary CancerNational Center for Cancer Care and Research, NCCCR, ALDoha, Qatar, Tel: 97444397800; E-mail: h.bugrein@yahoo.com Received February 08, 2017; Accepted March 16, 2017; Published March 24, 2017

Citation: Bujassoum SM, Bugrein HA, Al-Sulaiman R (2017) Genotype and Phenotype Correlation of Breast Cancer in BRCA Mutation Carriers and Noncarriers. J Cancer Sci Ther 9: 358-364. doi: 10.4172/1948-5956.1000442

Copyright: $\odot 2017$ Bujassoum SM, et al. This is an open-access article distributed under the terms of the Creative Commons Attribution License, which permits unrestricted use, distribution, and reproduction in any medium, provided the original author and source are credited. 
also been reported in cancers arising in BRCA genes mutation carriers [3]. BRCA1 is a tumor suppressor gene which was discovered in 1990 and was mapped to chromosome 17q21. BRCA1 contains 24 exons, encodes a protein of $-220 \mathrm{kDa}$, and composes of 1863 amino acids. BRCA1 has several cellular roles in DNA repair, cell-cycle regulation, transcriptional regulation and chromatin remodelling [4].

$B R C A 2$ is also a tumour suppressor gene and was discovered in 1994; it is localized on the long arm of chromosome 13. BRCA2 is a large gene with 27 exons that encode a protein of $380 \mathrm{kDa}$ and composes of 3418 amino acids [4]. BRCA2 function is restricted to DNA recombination and repair processes. Both BRCA1 and BRCA2 have an unusual large exon 11, non-coding exon 1 and a translational start site in exon 2. Their proteins are normally located in the nucleus and contain phosphorylated residues. BRCA1 and BRCA2 mutations are inherited in an autosomal dominant manner and they have incomplete penetrance. The most common types of mutations are small frame shift insertions or deletions, non-sense mutation or mutations affecting splice sites, resulting in deletion of complete or partial exons or insertion of intronic sequences, Large-scale rearrangements including insertions, deletions or duplications of more than $500 \mathrm{~kb}$ of DNA have also been identified [5].

The risk of individuals with BRCA1 or BRCA2 mutations of developing breast and ovarian cancers is approximately $45 \%$ to $87 \%$ for breast cancer by age of 70 and approximately $45 \%$ to $60 \%$ for ovarian cancer among BRCA1 mutation carriers and $11 \%$ to $35 \%$ among BRCA2 mutation carriers [6]. The most common form of histopathology of breast cancers in $B R C A$ gene mutation carriers are invasive ductal carcinoma (IDC) which is the most common histological subtype in both hereditary and sporadic breast cancer with some specific subtypes occurring more frequently such as medullary carcinoma. On the other hand, pre-invasive lesions ductal carcinoma in situ (DCIS) and lobular carcinoma in situ (LCIS) are less reported previously but in recent studies, it has been shown that premalignant lesions with remarkable lymph cytoplasmic infiltrate are detected in specimens of prophylactic mastectomy in $B R C A$ gene mutation carriers [7,4]. Studies have demonstrated that BRCA1-mutation carriers are more likely to be diagnosed with triple-negative (TN) breast cancer than non-carrier patients. Triple negative breast cancers are referred to estrogen receptor (ER) negative, progesterone receptor (PR) negative, and human epidermal growth factor receptor (HER2) negative tumors [8,9] and they have tendency to be more aggressive than other subtypes with higher nuclear grade and higher proliferative rate. In contrast, carriers of BRCA2 mutations seem to share similar pathologic characteristics with non-carrier patients $[10,11]$. In the literature reviews, it has been shown that most of the BRCA1 and BRCA2 mutations found in the breast and ovarian cancer families are predicted to truncate the protein product which lead to shortened and non-functional BRCA1 and $B R C A 2$ proteins; this may explain the apparently more aggressive biology of BRCA1 and BRCA2 breast cancers [12]. The most common types of mutations in $B R C A 1 / 2$ genes are small frame shift insertions or deletions, non-sense mutation or mutations affecting the splice sites which result in the deletion of complete or partial exons or insertion of intronic sequences. Loss of Heterozygosity $(\mathrm{LOH})$ of the wild type allele was also highly demonstrated in $B R C A$ related breast cancers. Identification of these mutations can be established by using multiplex ligation dependent probe amplification (MLPA) [4].

The aim of this retrospective study is to evaluate the impact of BRCA1 and BRCA2 pathogenic mutations on breast cancer aggressiveness, clinical, histological and molecular features by comparing the histogological and molecular information of breast cancer from BRCA positive patients (BRCA pathogenic mutation) and BRCA negative patients (no BRCA pathogenic mutations or variants of unknown significance).

\section{Patient and Methods}

The high-risk clinic for breast and ovarian cancers was established at the national center of cancer care and research (NCCCR) in the State of Qatar on 2013 by Dr. Salha Bujassoum, a senior medical oncologist, director of breast screening and the clinical leads of hereditary and high risk clinics in order to evaluate the risk of high risk patients of hereditary breast and ovarian cancer syndrome and to offer them risk reducing strategies if needed. This clinic has been recently expanded to include gastrointestinal and other malignancies. This clinic is supported by a board certified genetic counselor and a medical oncologist with experience in genetics [13].

The study was conducted in the State of Qatar at the National Centre of Cancer Care and Research; ethical approval was granted by the research ethics committee of the Hamad Medical Corporation (HMC-MRC) on 21 ${ }^{\text {st }}$ of March, 2015 (15031/15)

This is a retrospective cohort study that composed of 82 women diagnosed with breast cancer at ages of 50-year-old and younger who were evaluated and classified as high risk patients of carrying $B R C A$ gene mutations at the high-risk clinic at the national center of cancer care and research (NCCCR) in the State of Qatar between 2013-2015.

All patients who were classified as high risk and were eligible for genetic counselling assessment, were referred to the board certified genetic counsellor who was responsible for taking a detailed family history in the form of three generation pedigree in order to assess the possibility of a hereditary predisposition in the family. Based on the genetic counsellor's assessment, patients who were eligible for testing were offered testing for $B R C A-1 / 2$ full sequencing and multiple ligation dependent probe amplification (MLPA). Blood was collected in EDTA tubes and was sent for molecular testing. DNA was extracted using standard methods. Samples were analysed using direct DNA sequencing of all coding regions and intron-exon junctions and was evaluated for any deletions or duplications through both sequencing analysis and MLPA.

\section{Histopathology Review Study}

Tumor characteristics (coded according to the international classification of diseases for oncology, ICD-O 3) collected through retrospective review of histopathology reports. Samples included in the study (tumor blocks and slides) were reviewed again by designated breast pathologists to confirm and update the molecular profiling of the tumor tissue. Information on pathological stage, nodal involvement and histological type were collected from the histopathology reports. Nuclear grade was determined based on an assessment of tubule formation, nuclear pleomorphism and mitotic counts. This method of tumor graduation consists of scoring 1-3 for each factor. The grade is obtained by adding the three previous parameters and classifying the tumor as grade 1 (well differentiated or low-grade), grade 2 (moderately differentiated or intermediate-grade) and grade 3 (poorly differentiated or high-grade). This grading system has prognostic implications and the utility has been convincingly proved [14].

Hormone receptor status was labeled as positive when the fraction 
of cells stained by the corresponding immunoreactions was equal or higher than 1\%, according to American Society of Clinical Oncology Pathologist 2010, HER-2/neu status for cases of $1+, 2+$ or $3+$ on IHC analysis were further evaluated by fluorescent in situ hybridization for HER-2/neu amplification or over expression according to (ASOCOP), proliferation rate was considered high when $15 \%$ or more cells were stained by Ki- 67 antibody and lymphocytic infiltration and all of tumor biological characters correlated with BRCA1 and BRCA2 mutation type and with breast cancer tumor characteristics of breast cancer patients who had no pathogenic mutation or variant of unknown significant (VUS) in BRCA genes.

\section{Statistical Analysis}

Statistical analyses were performed by using the statistical package STATISTICA 11.0 version to perform data analysis and descriptive statistics. Two-sided fisher's exact test was used to assess the association between the type of receptors (ER, PR, and Her2), the tumor grade, histological subtype, lymph node involvement and nationality across two patients groups (BRCA negative, BRCA positive). A p-value of less than 0.05 using the two-tailed fisher's exact test was considered statistically significant. Comparison of age at diagnosis and the median tumor size across the BRCA groups was evaluated by using the Kruskalwallis exact test. A p value less than 0.05 was considered significant for accepting the hypothesis that a least two of the medians were significantly different from each other.

\section{Results}

The data analysis was carried out for the patients who tested positive or negative for BRCA1/2 genes. Patients who had VUS in the BRCA genes were excluded from this study. (82) Cases were included in this review: (22) were positive for BRCA1 mutations (9) were BRCA2 positive and (1) carried mutations in both BRCA1 and BRCA 2 and (50) patients had no pathogenic mutations in the BRCA genes and were considered as a control group (Figure 1). Median age at diagnosis of invasive breast cancer was 34 years in BRCA mutation carriers and the median age onset in non-carriers was 41 years.

Histopathological review study for breast cancer tumor of the (32) BRCA mutation carriers were compared with histopathology of breast cancer from the non BRCA carriers (50). The majority of invasive breast cancer arising in both BRCA1 and BRCA 2 carriers were Invasive Ductal Carcinoma (IDC) detected in $(30)(96.8 \%)(\mathrm{p}<0.05)$ of BRCA mutation carriers with medullary subtype and pushing margin in some BRCA1 tumors and areas of focal necrosis are more observed in BRCA1-related breast cancer than in BRCA2 and in sporadic breast cancers. Only 2 of BRCA carriers (3.1\%) had (DCIS). The overall frequency of high nuclear grade tumor (G3/3) in BRCA positive tumors $(75 \%)(\mathrm{p}<0.05)$ most of BRCA1 tumors and $(23 \%)$ in BRCA2 tumors and grade $2 / 3$ tumors ( G2) $25 \%$ most of grade 2 tumors are in BRCA2 positive, high proliferative rate Ki67 positive $56.2 \%$ in BRCA mutation carriers, low or negative in $25 \%$, Ecadherin was expressed positive in $53.1 \%$ of BRCA positive tumors and was undetermined in $46.9 \%$, Lymphocytic infiltration moderate to marked in $59 \%(\mathrm{p}<0.05)$ with some insufficiency in histological reports of determining lymphocytic infiltration in about $28 \%$ of the cases (Tables 1 and 2) (Figure 2).

Hormonal receptors ER, PR status for the BRCA mutation carriers and controls was obtained using immunohistochemical approaches ER negative, $\mathrm{PR}$ negative in (59.4\%) and ER positive, $\mathrm{PR}$ positive in $40.6 \%$ of cases, Her2 negative (90.6\%) and Her2 positive $9.3 \%$ in BRCA carriers. Triple-negative breast cancer were detected in 56.3\% in BRCA

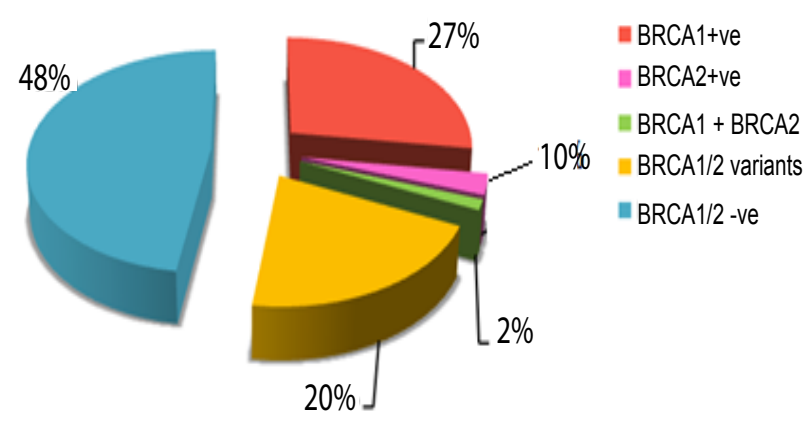

Figure 1: Results of genetic testing.

\begin{tabular}{|c|c|c|c|}
\hline \multirow[t]{2}{*}{ Marker } & $\begin{array}{l}\text { BRCA Mutation } \\
\text { carriers (32) }\end{array}$ & Non-carriers (50) & \multirow[t]{2}{*}{$P$ value } \\
\hline & N (\%) & $\mathrm{N}(\%)$ & \\
\hline \multicolumn{4}{|l|}{ ER } \\
\hline Positive & $13(40.6 \%)$ & $29(58.0 \%)$ & \multirow{3}{*}{$P<0.05$} \\
\hline Negative & $19(59.4 \%)$ & $15(30.0 \%)$ & \\
\hline Not determined & $0(0 \%)$ & $6(12.0 \%)$ & \\
\hline \multicolumn{4}{|l|}{ PR } \\
\hline Positive & $13(40.6 \%)$ & $27(54.0 \%)$ & \multirow{3}{*}{$P<0.05$} \\
\hline Negative & $19(59.4 \%)$ & $17(34.0 \%)$ & \\
\hline Not determined & $0(0 \%)$ & $6(12.0 \%)$ & \\
\hline \multicolumn{4}{|l|}{ Her2 } \\
\hline Positive & $3(9.4 \%)$ & $13(26.0 \%)$ & \multirow{3}{*}{$P<0.05$} \\
\hline Negative & $29(90.6 \%)$ & $30(60.0 \%)$ & \\
\hline Not determined & $0(\%)$ & $7(14.0 \%)$ & \\
\hline \multicolumn{4}{|l|}{$\mathrm{Ki}-67$} \\
\hline Positive & $18(56.2 \%)$ & $24(48 \%)$ & \multirow{3}{*}{$P>0.05$} \\
\hline Negative & $8(25 \%)$ & $12(24.0 \%)$ & \\
\hline Not determined & $6(18.8 \%)$ & $14(28.0 \%)$ & \\
\hline \multicolumn{4}{|l|}{ E cadherin } \\
\hline Positive & $17(53.1 \%)$ & $22(44.0 \%)$ & \multirow{3}{*}{$P<0.05$} \\
\hline Negative & $0(0 \%)$ & $20(40.0 \%)$ & \\
\hline Not determined & $15(46.9 \%)$ & $8(16.0 \%)$ & \\
\hline
\end{tabular}

Table 1: Comparison of immunohistochemical marker expression by BRCA status.

\begin{tabular}{|l|c|c|c|c|c|}
\hline \multirow{2}{*}{ Marker } & \multicolumn{2}{|c|}{$\begin{array}{c}\text { BRCA Mutation } \\
\text { carriers (23) }\end{array}$} & \multicolumn{2}{c|}{ Non-carriers (50) } & \multirow{2}{*}{ P value } \\
\cline { 2 - 5 } & $\mathrm{N}(+) / \mathrm{N}$ & Percentage & $\mathrm{N}(+) / \mathrm{N}$ & Percentage & \\
\hline Triple Negative & $18 / 32$ & $56.3 \%$ & $8 / 50$ & $16 \%$ & $\mathrm{P}<0.05$ \\
\hline Triple Positive & $3 / 32$ & $9.4 \%$ & $7 / 50$ & $14 \%$ & $\mathrm{P}>0.05$ \\
\hline $\begin{array}{l}\text { ER(+), PR(+) Her2 } \\
(-)\end{array}$ & $11 / 32$ & $34.4 \%$ & $22 / 50$ & $44 \%$ & $\mathrm{P}>0.05$ \\
\hline ER(-), PR(-) Her2 (+) & $0 / 32$ & $0 \%$ & $8 / 50$ & $16 \%$ & $\mathrm{P}<0.05$ \\
\hline
\end{tabular}

Table 2: Breast cancer characteristics by BRCA status.

mutation carrier's tumors $(\mathrm{p}<0.05)$, Triple positive i.e. positive for $\mathrm{ER}$, PR and Her2 (9.4\%) in BRCA positive and ER+ve PR+ve Her2 -ve (34.4\%), no ERPR -ve HER2+ve detected in BRCA carriers tumors. Disease Stage was carried out according to AJCC staging manual 

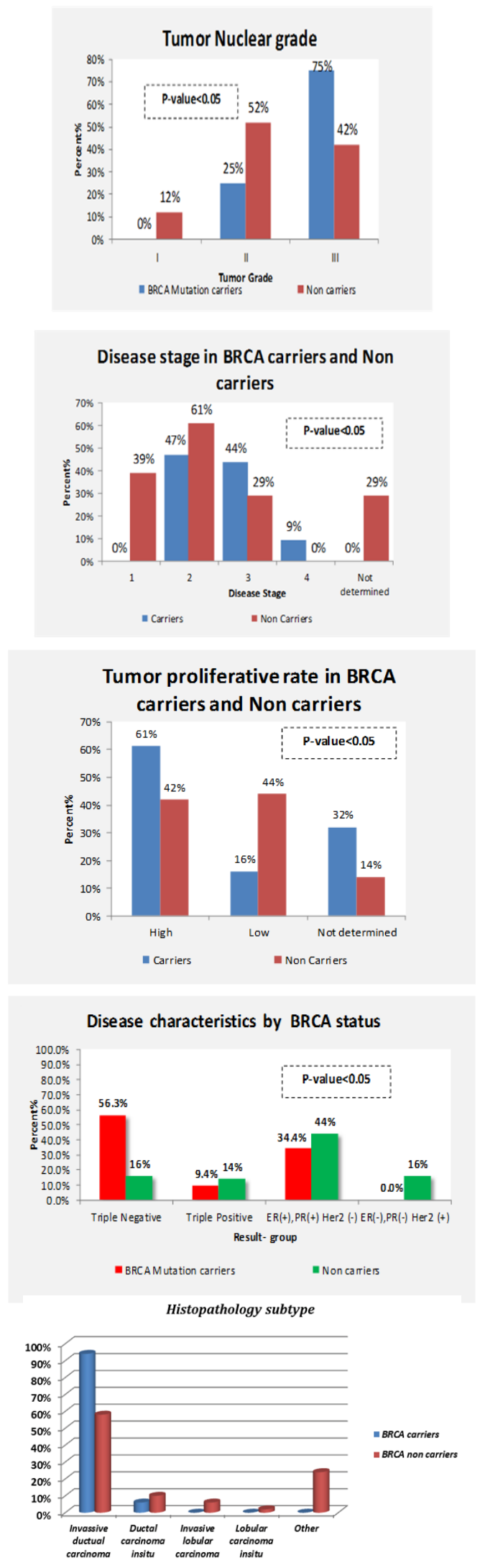

Figure 2: Tumor nuclear grade, disease stage and characteristics between BRCA carriers and non-carriers.
BRCA positive diagnosed at stage II $47 \%$, stage III $44 \%$, stage IV $9 \%$ in advanced stage (Tables 1 and 2) (Figure 2).

The most common breast tumor histopathology in non-carriers control group was IDC (58\%), however, other non-carriers exhibited other histological types detected with various percent, DCIS (10\%), invasive lobular carcinoma (ILC) (6\%), Lobular carcinoma in situ (LCI) $2 \%$ with a p-value $(\mathrm{p}<0.05)$. high tumor grade G3 42\%, G2 36\%, G1 $6 \%$, and $31 \%$. due to difficulties in the available tumor tissue, Ecadherin positive in $44 \%$ and negative in $20 \%$ of matched control and undetermined in $16 \%$. ER negative $30 \%$, PR negative $34 \%$ of non-carriers and Her2 negative (26\%), and Her2 positive (60\%) undetermined for 6 cases. And 17\% in BRCA negative tumors, tumors and $(18 \%)(\mathrm{p}>0.05)$ of non-carriers, ER positive (58\%) of non-carriers. And PR positive (54\%), and disease stage on diagnosis in the matched control non carriers, stage I $39 \%$, stage II $61 \%$, stag III $29 \%$, and no cases in stage IV $(\mathrm{p}<0.05)$ (Table 3 and Figure 2).

In our study, we also found that the most common detected mutations were small frame shift deletion or insertion in the BRCA1 and $B R C A 2$ genes and most BRCA positive patients had mutations in the $B R C A 1$ gene (Table 4 ). (Percentages calculated by column ${ }^{*} \mathrm{P}$ value from kruskall-wallis exact test $\mathrm{P}$ value from fisher's exact).

\section{Discussion}

The goal of this study was to explore the effect of $B R C A 1$ or $B R C A$ 2 germline mutations on breast cancer aggressiveness, clinical and histological features. A retrospective study was conducted to study breast cancer cases that were evaluated to be high risk for carrying BRCA mutations at the hereditary breast and ovarian cancer clinic at the NCCCR in the State of Qatar from 2013 to 2015. Review of medical records was conducted to determine the clinical characteristics, the molecular results of BRCA testing and the tumor characteristics from the histopathology reports in addition to a new review of the tumor blocks to update the molecular data of those patients. A total of 82 breast cancer patients were included in the analyses (32 patients with $B R C A$ gene pathogenic mutations and 50 patients with no pathogenic mutations or VUS's in the BRCA genes). We compared the histological, molecular status and clinical variables of the 32 BRCA gene mutation carriers to the histopathological and molecular characteristics of the 50 patients with no pathogenic mutations or variants of unknown significant (VUS) in either BRCA1 or BRCA2 genes [15].

The 82 patients that were included in this retrospective study had early onset breast cancer (50 years of age and younger). A total of 22 patients were found to have BRCA1 pathogenic mutations, (9) patients had BRCA2 pathogenic mutations and (1) patient carried pathogenic mutations in both BRCA1 and BRCA2 genes.

Our results suggest that BRCA mutations were detected among women of younger ages and that the majority of BRCA associated breast cancers were invasive ductal carcinoma (IDC) detected with high nuclear grade $3 / 3$, have a higher number of mitosis and show a high frequency of necrotic areas, and a higher proliferative rate and lymphocytic infiltration which points toward a more aggressive tumor type. In addition, we found that most of the BRCA associated breast cancers were triple negatives and were stage II when compared to those women with no pathogenic mutations who most of them had stage I and most of them had IDC with ER PR positive Her2 negative status and with less aggressive profile.

We also found that most of the BRCA1 breast carcinomas have the basal cell phenotype, highly proliferating, estrogen receptor 


\begin{tabular}{|c|c|c|c|}
\hline Parameter & BRCA Mutation Carriers N (\%) & Non-carriers $\mathrm{N}(\%)$ & $P$ value \\
\hline Total & $32(39 \%)$ & $50(61 \%$ & \\
\hline \multicolumn{4}{|c|}{ Tumor Grade } \\
\hline 1 & $0(0 \%)$ & $6(12 \%)$ & \multirow{4}{*}{$p+<0.05$} \\
\hline II & $8(25.0 \%)$ & $17(34 \%)$ & \\
\hline III & $24(75 \%)$ & $21(42 \%)$ & \\
\hline Not determined & $0(0 \%)$ & $6(12 \%)$ & \\
\hline \multicolumn{4}{|c|}{ Age of disease onset } \\
\hline under age 50 & $30(94 \%)$ & $44(88 \%)$ & \multirow{5}{*}{${ }^{*} \mathrm{P}>0.05$} \\
\hline age 50 or older & $2(6 \%)$ & $6(12 \%)$ & \\
\hline Mean SD, years & $40.56+8.82$ & $43.32+7.96$ & \\
\hline Median, years & 34 & 41 & \\
\hline Range, years & $(23-63)$ & $(30-68)$ & \\
\hline \multicolumn{4}{|c|}{ Tumor size } \\
\hline Mean \pm SD & $3.82 \pm 1.45$ & $2.98 \pm 1.03$ & \multirow{3}{*}{$P^{*}<0.05$} \\
\hline Median & 4 & 2 & \\
\hline Range & $(0.4-7)$ & $(0.4-6)$ & \\
\hline \multicolumn{4}{|c|}{ Histological subtype } \\
\hline Invasive ductual carcinoma & $30(96.8 \%)$ & $29(58 \%)$ & \multirow{5}{*}{$p+>0.05$} \\
\hline Ductal carcinoma in situ & $2(3.1 \%)$ & $5(10 \%)$ & \\
\hline Invasive lobular carcinoma & $0(0 \%)$ & $3(6 \%)$ & \\
\hline Lobular carcinoma in situ & $0(0 \%)$ & $1(2 \%)$ & \\
\hline Other & $0(0 \%)$ & $12(24 \%)$ & \\
\hline \multicolumn{4}{|c|}{ Lymphocytic infiltrate } \\
\hline Moderate/marked & $19(59 \%)$ & $21(42 \%)$ & \multirow{3}{*}{$\mathrm{P}+<0.05$} \\
\hline None/mild & $4(13 \%)$ & $22(44 \%)$ & \\
\hline Not determined & $9(28 \%)$ & $7(14 \%)$ & \\
\hline \multicolumn{4}{|c|}{ Stage of disease } \\
\hline 1 & $0(0 \%)$ & $17(34 \%)$ & \multirow{5}{*}{$\mathrm{P}+<0.05$} \\
\hline 2 & $15(47 \%)$ & $17(34 \%)$ & \\
\hline 3 & $14(44 \%)$ & $6(12 \%)$ & \\
\hline 4 & $3(9 \%)$ & $0(00 \%)$ & \\
\hline Not determined & $0(0 \%)$ & $10(20 \%)$ & \\
\hline
\end{tabular}

progesterone receptor and HER2-negative (Triple negative breast carcinomas), characterized by the expression of basal like. BRCA2 tumors are of higher tumor grade than sporadic age matched controls, Luminal B phenotype but tend to be estrogen receptor and progesterone receptor positive, and non-BRCA1/2 sporadic tumors are more phenotypically heterogeneous and have phenotypic similarities to BRCA2 tumors, but tend to be of lower tumor grade and proliferation index and most often of the ER+ luminal subtypes [16].

We sought to validate the impact of BRCA genes mutation on breast cancer biology. More aggressive tumors were detected in BRCA carriers with more advanced disease stage when compared with a matched young age group of BRCA non-carrier. In this study, we also showed that the most common type of BRCA mutation is small frame shift deletion or insertion that causes protein truncation $[17,18]$ Most BRCA carriers in this study had pathogenic mutations in the BRCA1 gene with most of them being frameshift mutations.

\section{Conclusion}

We identified genotypic and pathologic characteristics of breast cancer tumors in a cohort of women with BRCA-positive and BRCAnegative genetic testing. Heterogeneous pathogenic BRCA mutation was detected with higher incidence in younger age with the most common mutations being frame shift mutation in the BRCAl gene.
Most of the BRCA1 related breast cancers were triple negative than the BRCA 2 and BRCA1/2 negative associated breast cancers. In addition, BRCA1 related breast cancers were poorly differentiated and had a higher nuclear grade and were of more advanced disease stage than BRCA2. The prognosis of triple Negative tumors is very poor, not only because these tumors seem to be more aggressive than other breast cancers but the endocrine and anti-HER2 therapies are ineffective, leaving chemotherapy as the only treatment option available.

Further analysis is needed to determine clinical outcomes of BRCA positive breast cancers in regards to response to chemotherapy, local and systemic relapse overall survival in comparison to BRCA negative breast cancers. We will continue to build up our data base to better characterize our hereditary breast cancer cases at clinical and molecular levels which could help to a better understand the morphological, immunohistochemical and molecular characteristics of BRCA1 BRCA2 hereditary breast cancers which could offer clues for diagnosis and new therapeutic approaches $[19,20]$.

More research is also needed to study the variants of uncertain significance found in the $B R C A 1 / 2$ genes which could impact the molecular signatures of breast cancers. This could help in clarifying the significance of VUS's on tumor biology and future risks of cancer which in turn could help in the classifications of these variants. 
Citation: Bujassoum SM, Bugrein HA, Al-Sulaiman R (2017) Genotype and Phenotype Correlation of Breast Cancer in BRCA Mutation Carriers and Non-carriers. J Cancer Sci Ther 9: 358-364. doi: 10.4172/1948-5956.1000442

\begin{tabular}{|c|c|c|c|c|c|c|c|c|c|c|c|}
\hline Case & Age & History & Nuclear Grade & ER & PR & Her2 & Ki67 & Ecadherin & $B R C A$ gene & Mutation & Type \\
\hline 1 & 39 & DCIS & 3 & + & + & - & ND & ND & $B R C A 1$ & c. $2281 G>T$ p.E761X & Frameshift \\
\hline 2 & 28 & IDC & 3 & - & - & - & ND & ND & $B R C A 1$ & c.68_69del p.Glu23va1fs*17) & Frameshift \\
\hline 3 & 49 & IDC & 2 & + & + & - & - & + & $B R C A 2$ & c.1813dupc pl1e605Asnfs*11) & Frameshift \\
\hline 4 & 29 & IDC & 3 & - & - & - & ND & ND & $B R C A 1$ & c.5096G>A p.R1699Q & Frameshift \\
\hline 5 & 46 & IDC & 3 & - & - & - & + & ND & $B R C A 1$ & c.4136_4137 de1CT p.S1379* & Nonsense \\
\hline 6 & 42 & IDC & 2 & - & - & - & ND & ND & $B R C A 1$ & c.4787C>A p.S1596X & Frameshift \\
\hline 7 & 29 & IDC & 2 & - & - & - & ND & ND & $B R C A 1$ & c. 4787 C>A p.S1596X & Frameshift \\
\hline 8 & 41 & IDC & 3 & - & - & - & - & ND & $B R C A 1$ & $\begin{array}{l}\text { c.4065_4068de1TCAAp. } \\
\text { N1355fs aka 4184de14 }\end{array}$ & Frameshift \\
\hline \multirow{2}{*}{9} & \multirow{2}{*}{43} & \multirow{2}{*}{ IDC } & \multirow{2}{*}{2} & \multirow{2}{*}{-} & \multirow{2}{*}{-} & \multirow{2}{*}{ - } & \multirow{2}{*}{+} & \multirow{2}{*}{ ND } & \multirow{2}{*}{$B R C A 1 / 2$} & c. $211 \mathrm{~A}>\mathrm{G}$ p.Arg71G1y & \multirow{2}{*}{ Frameshift } \\
\hline & & & & & & & & & & c.5640T>GP.Asn1880Lys & \\
\hline 10 & 27 & IDC & 2 & + & + & - & + & ND & $B R C A 2$ & c. $440 A>G$ p.G $\ln 147$ Arg & Frameshift \\
\hline 11 & 51 & IDC & 2 & + & + & + & + & + & $B R C A 1$ & c. 4787 C >A p. Ser $1596^{*}$ & Frameshift \\
\hline 12 & 41 & IDC & 3 & - & - & - & + & + & $B R C A 1$ & c. $4787 \mathrm{C}>\mathrm{A}$ for $p$. ser $1596^{*}$ & Frameshift \\
\hline 13 & 23 & IDC & 3 & - & - & - & + & ND & $B R C A 1$ & c. $4850 C>A$ p. $S 1617 X$ & Frameshift \\
\hline 14 & 36 & IDC & 3 & + & + & - & - & + & $B R C A 2$ & c. 9027 delT p. His3010l1efs ${ }^{*} 18$ & Frameshift \\
\hline 15 & 35 & IDC & 3 & + & + & - & - & + & $B R C A 2$ & c. 9027 delT p. His3010l1efs ${ }^{*} 18$ & Frameshift \\
\hline 16 & 38 & IDC & 2 & + & + & - & - & ND & $B R C A 1 / 2$ & $\begin{array}{l}1456 \mathrm{~T}>\mathrm{C} \text { p.Phe486Leu } \\
\text { c.1648A }>\mathrm{C} \text { p.Asn550His } \\
\text { c.536A }>\text { G p.Tyr179Cys } \\
\text { c.7007G }>\text { A p. Arg2336His }\end{array}$ & Frameshift \\
\hline 17 & 39 & IDC & 3 & - & - & - & + & + & $B R C A 1$ & c.4787C>A p.Ser1596* & Frameshift \\
\hline 18 & 37 & IDC & 3 & - & - & - & + & ND & $B R C A 1$ & c. 679 G>T p. Glu2227 & Nonsense \\
\hline 19 & 43 & IDC & 2 & + & + & - & - & + & $B R C A 1$ & $R 71 G(330 A>G)$ & Frameshift \\
\hline 20 & 33 & IDC & 3 & - & - & - & - & + & $B R C A 1$ & c. $4986+6 \mathrm{~T}>\mathrm{C}$ & Frameshift \\
\hline 21 & 53 & IDC & 3 & - & - & - & ND & ND & $B R C A 1$ & c.66dupA (p.G1u23Argfs¹8) & Frameshift \\
\hline 22 & 32 & IDC & 3 & - & - & - & ND & ND & $B R C A 1$ & c04850C>A p.S1617X & Frameshift \\
\hline 23 & 38 & IDC & 2 & + & + & + & - & ND & BRCA2 & c.5640T>G p.Asn1880Lys & Frameshift \\
\hline 24 & 29 & IDC & 3 & - & - & - & + & + & $B R C A 1$ & c. $2158 \mathrm{G}>\mathrm{T}$ p.Glu720* & Frameshift \\
\hline 25 & 36 & IDC & 3 & + & + & - & + & + & $B R C A 2$ & $\begin{array}{c}\text { c.4111C>T,P. } \\
\text { gLN1371Ter,rs80358659 }\end{array}$ & Nonsense \\
\hline 26 & 34 & IDC & 3 & - & - & - & + & + & $B R C A 1$ & c. 4787 C>A p.Ser $1596^{*}$ & Nonsense \\
\hline 27 & 36 & IDC & 2 & - & - & - & + & + & $B R C A 1$ & c.5266dupC (p.Gln175Profs) & Frameshift \\
\hline 28 & 33 & DCIS & 2 & + & + & - & - & + & $B R C A 1$ & c.5036C>A, p.Thr1679Asn & Missense \\
\hline 29 & 29 & IDC & 3 & + & + & - & + & + & $B R C A 2$ & $\begin{array}{l}\text { c.6405_6409delCTTAA(p. } \\
\text { Asn2135Lysfs),rs80359585 }\end{array}$ & Frameshift \\
\hline 30 & 28 & IDC & 3 & - & - & - & + & + & $B R C A 1$ & $\begin{array}{c}\text { c.3436_3439ITGTT, } \\
\text { (p.Cys1146_eufs),rs397509067 }\end{array}$ & Frameshift \\
\hline 31 & 64 & IDC & 2 & + & + & + & + & + & $B R C A 1$ & $\begin{array}{l}\text { c.8053dupA, p.Thr2685fs, } \\
\text { rs397507959 }\end{array}$ & Frameshift \\
\hline 32 & 56 & IDC & 2 & + & + & - & & & $B R C A 2$ & c.6322C>T(p.Arg2108Cys) & Missense \\
\hline
\end{tabular}

Table 4: Mutations types in the BRCA1 and BRCA2 genes among mutations carriers.

\section{References}

1. Larsen MJ, Thomassen M, Gerdes AM, Kruse TA (2014) Hereditary breas cancer: Clinical, pathological and molecular characteristics. Breast Cancer (Auckl) 8: 145-155.

2. BRCA1 and BRCA2: Prevalence and risks for breast and ovarian cancer.

3. American Cancer Society: Cancer Facts and Figures 2014. Atlanta, Ga: American Cancer Society.

4. Karp J, Osin VB (1998) Advances in cancer research. An Elsevier Science imprint, California, USA.

5. Grigorijs P, Arvids I, Andris G, Signe S, Santa R, et al. (2011) Genotypephenotype correlations among BRCA14153delA and 5382insC mutation carriers from Latvia. BMC Medical Genetics 12: 147.

6. Genetics of Breast and Ovarian Cancer National Cancer

7. Futreal PA, Liu Q, Shattuck-Eidens D, Cochran C, Harshman K, et al. (1994) BRCA1 mutations in primary breast and ovarian carcinomas. Science 266: 120-122.

8. Brody LC, Biesecker BB (1998) Breast cancer susceptibility genes. BRCA1 and BRCA2. Medicine (Baltimore) 77(3): 208-226.
9. Breast cancer in Arab populations: molecular characteristicsand disease management implications Lotfi Chouchane, Hammouda Boussen, Konduru S R Sastry Qatar cancer registry for breast cancer registry data on 2009 -2014.

10. King MC, Marks JH, Mandell JB (2003) Breast and ovarian cancer risks due to inherited mutations in BRCA1 and BRCA2. Science 302(5645): 643-646.

11. Mangia A, Malfettone A, Simone G, Darvishian F (2011) Old and new concepts in histopathological characterization of familial breast cancer. Ann Oncol 22(suppl 1): i24-i30.

12. Miki Y, Swensen J, Shattuck-Eidens D, Futreal P, Harshman K, et al. (1994) A strong candidate for the breast and ovarian cancer susceptibility gene BRCA1. Science 266: 66-71.

13. Loman N, Johannsson O, Bendahl PO, Borg A, Fernö M, et al. (1998) Steroid receptors in hereditary breast carcinomas associated with BRCA1 or BRCA2 mutations or unknown susceptibility genes. Cancer 83(2): 310-319.

14. WF Li, Hu Z, Rao NY, Song CG, Zhang B, et al. (2007) The prevalence of BRCA1 and BRCA2 germline mutations in high-risk breast cancer patients of Chinese Han nationality: two recurrent mutations were identified. Cancer Res Treat 110(1): 99-109. 
Citation: Bujassoum SM, Bugrein HA, Al-Sulaiman R (2017) Genotype and Phenotype Correlation of Breast Cancer in BRCA Mutation Carriers and Non-carriers. J Cancer Sci Ther 9: 358-364. doi: 10.4172/1948-5956.1000442

15. Tung N, Wang Y, Collins LC, Kaplan J, Li H, et al. (2010) Estrogen receptor positive breast cancers in BRCA1 mutation carriers: clinical risk factors and pathologic features. Breast Cancer Res 12(1): R12.

16. van der Groep P, van der Wall E, van Diest PJ (2011) Pathology of hereditary breast cancer. Cell oncol 34(2): 71-88.

17. Sana M, Irshad S (2012) A review on breast cancer biomarkers BRCA1 and BRCA2. Res Cancer Tumor 1(1): 1-4.
18. Martin JL, Thomassen M, Anne-Marie G, Torben AK (2014) Hereditary breast cancer: Clinical, pathological and molecular characteristics. Breast Cancer (Auckl) 8: 145-155.

19. Tan DS, Marchio C, Reis-Filho JS (2008) Hereditary breast cancer: From molecular pathology to tailored therapies. J Clin Pathol 61(10): 1073-1082

20. Honrado E, Benítez J, Palacios J (2005) The molecular pathology of hereditary breast cancer: Genetic testing and therapeutic implications. Modern Patho 18(10): 1305-1320. 